9th A. Friedmann International Seminar and

3rd Casimir Symposium 2015

International Journal of Modern Physics: Conference Series

Vol. 41 (2016) 1660124 (8 pages)

(C) The Author(s)

DOI: $10.1142 /$ S2010194516601241

\title{
Gravitational collapse of Vaidya spacetime
}

\author{
Vitalii Vertogradov \\ Physics Department, Herzen State Pedagogical University of Russia, \\ Moika 48, St. Petersburg 191186, Russia \\ vitalii.vertogradov@yandex.ru \\ Received 18 September 2015 \\ Revised 1 October 2015 \\ Published 18 March 2016
}

\begin{abstract}
The gravitational collapse of generalized Vaidya spacetime is considered. It is known that the endstate of gravitational collapse, as to whether a black hole or a naked singularity is formed, depends on the mass function $M(v, r)$. Here we give conditions for the mass function which corresponds to the equation of the state $P=\alpha \rho$ where $\alpha \in\left(0, \frac{1}{3}\right]$ and according to these conditions we obtain either a black hole or a naked singularity at the endstate of gravitational collapse. Also we give conditions for the mass function when the singularity is gravitationally strong.
\end{abstract}

Keywords: Gravitational collapse; naked singularity; strength of singularity; black hole; Vaidya spacetime.

PACS numbers: 04.70.-s, 04.70.Bw, 97.60.Lf

\section{Introduction}

In recent articles of A. A. Grib, Yu. V. Pavlov and V. D. Vertogradov ${ }^{1,2}$ properties of geodesics for particles with negative energy or Penrose geodesics in Kerr metric have been studied. It has been shown that all such geodesics appear in the ergosphere from a region inside the gravitational radius. However Kerr black holes are eternal ones. In real case one needs to consider the question about the gravitational collapse to learn the nature of Penrose geodesics. One of the opportunities for existence of such geodesics is the naked singularity formation during the gravitational collapse.

In the beginning we decided to consider the simplest case of gravitational collapse - the case of spherically symmetric collapse, in particular — the case of gravitational collapse of generalized Vaidya spacetime which will be treated in this article.

This is an Open Access article published by World Scientific Publishing Company. It is distributed under the terms of the Creative Commons Attribution 4.0 (CC-BY) License. Further distribution of this work is permitted, provided the original work is properly cited. 
It is worth mentioning that Papapetrou ${ }^{3}$ showed that Vaidya metric (also known as the radiating Schwarzschild spacetime) breaks the cosmic censorship principle. So Vaidya metric is the earliest counterexample of cosmic censorship violation.

$\mathrm{Joshi}^{4}$ showed that result of gravitational collapse, as to whether a black hole or a naked singularity is formed, depends on the initial data. In the resent paper ${ }^{5}$ it has been shown that the endstate of gravitational collapse of generalized Vaidya spacetime depends on the mass function $M(v, r)$. Also in Ref. 6 the attempt has been made to show that the result of gravitational collapse of Vaidya-DeSitter spacetime is the naked singularity. However in this papers the question about the apparent horizon formation has not been considered. But in the case of Vaidya-DeSitter spacetime the time of the apparent horizon formation is less than the time of the singularity formation, so the result of such collapse is the black hole.

We consider the gravitational collapse of thin radiating shells. The first shell collapses down at the central singularity at $r=v=0$ where $M(0,0)=0$. During the collapse of other shells the mass function is growing and when the last shell collapses down then the mass function becomes well-known Schwarzschild mass. Also we are only interested in shell-focusing singularities. Shell-crossing singularities are gravitationally weak and we are not interested in them. If there is a family of nonspacelike future-directed geodesics which originate at the central singularity in past and the time of singularity formation is less than the time of the apparent horizon formation then the result of such gravitational collapse is the naked singularity. If there is no such a family of geodesics or the time of the apparent horizon formation is less than the time of singularity formation then the result is the black hole. It is worth mentioning that here we consider only locally naked singularities. It means that the apparent horizon is formed earlier than the geodesic crosses the last collapsing thin shell. If it is not so then the singularity is global naked one. But here we won't consider global naked singularities.

In this paper we give conditions for the mass function and corresponding to these conditions we obtain either a naked singularity or a black hole as a result of gravitational collapse. We consider the matter which satisfies the equation of the state $P=\alpha \rho$ where $\alpha \in\left(0, \frac{1}{3}\right]$. Also we give conditions for mass function and corresponding to these conditions we obtain the gravitationally strong singularity. If we follow Tipler definition which was given in the paper: ${ }^{7}$ a singularity is termed to be gravitationally strong or simply strong if it destroys by stretching or crushing any object which falls into it. If it does not destroy any object this way then the singularity is termed to be gravitationally weak.

In Sec. 2 we give basic information about generalized Vaidya spacetime. In Sec. 3 we present conditions for the mass function when we obtain either a naked singularity or a black hole. In Sec. 4 we give conditions for the mass function when the singularity is gravitationally strong.

The system units $G=c=1$ will be used in this paper. Dash and dot denote partial derivatives $\frac{d}{d r}, \frac{d}{d v}$ respectively. Values $g_{\alpha \beta}, \Gamma_{\beta \gamma}^{\alpha}, R_{\alpha \beta}$ are metric, Christoffel and Ricci tensors components respectively. Greek letters are equal to 0,1,2,3. 


\section{Generalized Vaidya Spacetime}

Generalized Vaidya spacetime corresponds to the combination of two matter fields I and II types and in general case is given by: ${ }^{8}$

$$
\begin{array}{r}
d s^{2}=-e^{2 \psi(v, r)}\left(1-\frac{2 M(v, r)}{r}\right) d v^{2}+2 \varepsilon e^{\psi(v, r)} d v d r+r^{2} d \omega^{2}, \\
d \omega^{2}=d \theta^{2}+\sin ^{2}(\theta) d \varphi^{2},
\end{array}
$$

here $M(v, r)$ - the mass function depending on coordinates $r$ and $v$ which corresponds to advanced/retarded time, $\varepsilon= \pm 1$ — ingoing/outgoing radiating thin shells respectively.

So we are interested in gravitational collapse then we put $\varepsilon=+1$. Also with suitable choice of coordinates we can put $\psi(v, r)=0$. So (1) now has the form:

$$
d s^{2}=-\left(1-\frac{2 M(v, r)}{r}\right) d v^{2}+2 \varepsilon d v d r+r^{2} d \omega^{2} .
$$

Now let us write down non vanishing covariant and contravariant metric components:

$$
\begin{aligned}
& g_{00}=-\left(1-\frac{2 M(v, r)}{r}\right), g_{01}=1, g_{22}=r^{2}, g_{33}=r^{2} \sin ^{2}(\theta) . \\
& g^{01}=1, g^{11}=\left(1-\frac{2 M(v, r)}{r}\right), g^{22}=\frac{1}{r^{2}}, g^{33}=\frac{1}{r^{2} \sin ^{2}(\theta)} .
\end{aligned}
$$

Now we can write down non-vanishing Christoffel components:

$$
\begin{array}{r}
\Gamma_{00}^{0}=\frac{M-m^{\prime} r}{r^{2}}, \\
\Gamma_{22}^{0}=-r, \\
\Gamma_{33}^{0}=-r \sin ^{2}(\theta), \\
\Gamma_{00}^{1}=\frac{\left(1-\frac{2 M}{r}\right)\left(M-M^{\prime} r\right)+\dot{M} r}{r^{2}}, \\
\Gamma_{10}^{1}=-\gamma_{00}^{0}, \Gamma_{22}^{1}=2 M-r, \\
\Gamma_{33}^{1}=\sin ^{2}(\theta)(2 M-r), \\
\Gamma_{12}^{2}=\gamma_{13}^{3}=\frac{1}{r}, \\
\Gamma_{33}^{2}=-\sin (\theta) \cos (\theta), \\
\Gamma_{23}^{3}=\operatorname{ctg}(\theta) .
\end{array}
$$

Now let us write down non-vanishing Ricci components:

$$
R_{01}=\frac{M^{\prime \prime}}{r},
$$




$$
\begin{array}{r}
R_{00}=\frac{(2 M-r) M^{\prime \prime}+2 \dot{M}}{r^{2}}, \\
R_{22}=2 M, \\
R_{33}=\sin ^{2}(\theta) 2 M^{\prime} .
\end{array}
$$

We can write down the energy momentum tensor in the following form:

$$
T_{\mu \nu}=T_{\mu \nu}^{(n)}+T_{\mu \nu}^{(m)},
$$

where the first term corresponds to the matter field I type and the other one corresponds to the matter field II type.

Now let us write down the expression of the energy momentum tensor:

$$
\begin{array}{r}
T_{\mu \nu}^{(n)}=\mu L_{\mu} L_{\nu}, \\
T_{\mu \nu}^{(m)}=(\rho+P)\left(L_{\mu} N_{\nu}+L_{\nu} N_{\mu}\right), \\
\mu=\frac{2 \dot{M}}{r^{2}}, \\
\rho=\frac{2 M^{\prime}}{r^{2}}, \\
P=-\frac{M^{\prime \prime}}{r}, \\
L_{\mu}=\delta_{\mu}^{0}, \\
N_{\mu}=\frac{1}{2}\left(1-\frac{2 M}{r}\right) \delta_{\mu}^{0}-\varepsilon \delta_{\mu}^{1}, \\
L_{\mu} L^{\mu}=N_{\mu} N^{\mu}=0, \\
L_{\mu} N^{\mu}=-1 .
\end{array}
$$

Here $P$ - pressure, $\rho$ - density and $L, N$ - two null vectors.

This model must be physically reasonable so the energy momentum tensor should satisfies weak, strong and dominant energy conditions. It means that $\rho$ must be positive and for any non-spacelike vector $v^{\alpha}$ :

$$
T_{\alpha \beta} v^{\alpha} v^{\beta}>0
$$

and the vector $T_{\alpha \beta} v^{\alpha}$ must be timelike.

Strong and weak energy conditions demand:

$$
\mu \geq 0, \rho \geq 0, P \geq 0 \text {. }
$$

The dominant energy condition imposes following conditions on the energy momentum tensor:

$$
\mu \geq 0, \rho \geq P \geq 0
$$


Also let us introduce following equations and notation. The equation of radial null geodesic has form:

$$
\frac{d v}{d r}=\frac{2 r}{r-2 M}
$$

The existence of a family of non-spacelike future-directed geodesics which originate at the central singularity in past is defined by sign $\frac{d v}{d r}$. If the sign is " " "' then such family exists if the sign is " _ " then such family does not exist. Also let us introduce the following notation $X_{0}$ as:

$$
x_{0}=\lim _{v \rightarrow 0, r \rightarrow 0} \frac{v}{r} \text {. }
$$

\section{The Endstate of Gravitational Collapse}

In the beginning let us consider the simplest case when the equation of the state is:

$$
P=\frac{\rho}{3} \text {. }
$$

Then if we use (8) then we obtain the mass function:

$$
\begin{array}{r}
3 M^{\prime \prime}(v, r) r+2 M^{\prime}(v, r)=0, \\
M(v, r)=C(v)+D(v) r^{\frac{1}{3}} .
\end{array}
$$

Pressure and density are given by:

$$
\begin{aligned}
& \rho=\frac{2}{3} \frac{D(v)}{r^{\frac{8}{3}}}, \\
& P=\frac{2}{9} \frac{D(v)}{r^{\frac{8}{3}}} .
\end{aligned}
$$

Strong, weak and dominant energy conditions demand:

$$
\begin{aligned}
D(v) & \geq 0, \\
\dot{C}(v)+\dot{D}(v) r^{\frac{1}{3}} & \geq 0, \\
C(v) & \geq 0 .
\end{aligned}
$$

Also the condition $M(0,0)=0$ demands:

$$
C(0)=0 .
$$

The equation of the apparent horizon is given by:

$$
r=2 C(v)+2 D(v) r^{\frac{1}{3}} .
$$

Now we can see that when $v=0$ is the time of the singularity formation then the equation of the apparent horizon is given by:

$$
r=2 D(0) r^{\frac{1}{3}}
$$


If $D(0)>0$ then $r>0$ and we have that the time of the apparent horizon formation is less than the time of singularity formation and in this case we have a black hole as a result of gravitational collapse. Hence naked singularity is formed only then when $D(0)=0$.

Now let us consider the question about existence of a family of non-spacelike future-directed geodesics which originate at central singularity $r=v=0$ in the past. Let us substitute the mass function (15) into (12):

$$
\frac{d v}{d r}=\frac{2 r}{r-2\left(C(v)+D(v) r^{\frac{1}{3}}\right)} .
$$

Now if we consider the limit at $r \rightarrow 0, v \rightarrow 0$ in (21) we obtain the following conditions for mass function and according to these conditions we have naked singularity as a result of gravitational collapse:

$$
\begin{aligned}
D(0) & =0, \\
\lim _{v \rightarrow 0, r \rightarrow 0} \frac{C(v)}{r}=a \geq 0, & \\
\lim _{v \rightarrow 0, r \rightarrow 0} \frac{D(v)}{r^{\frac{2}{3}}}=b & \geq 0, \\
2 a+2 b & <1,
\end{aligned}
$$

where $a, b$ - arbitrary constants.

So when conditions (22) are satisfied then we have naked singularity as a result of collapse.

Now let us consider more general case when the equation of the state is given by:

$$
P=\alpha \rho, \alpha \in\left(0, \frac{1}{3}\right) .
$$

Here we use (8) and obtain the mass function:

$$
M(v, r)=C(v)+D(v) r^{1-2 \alpha} .
$$

Now if we use (24) we obtain expression for the pressure and the density:

$$
\begin{array}{r}
P=2 \alpha(1-2 \alpha) \frac{D(v)}{r^{2 \alpha+2}}, \\
\rho=2(1-2 \alpha) \frac{D(v)}{r^{2 \alpha+2}}, \\
\mu=2 \frac{\dot{C}(v)+\dot{D}(v) r^{1-2 \alpha}}{r^{2}} .
\end{array}
$$

We can easily see that in general case strong, weak and dominant energy conditions impose the same requirements as in case when the equation of state is $P=\frac{1}{3} \rho$. Also it is not difficult to show that conditions for the mass function are the same like in previous case. 
So we have given conditions for the mass function when the equation of the state is $P=\alpha \rho, \alpha \in\left(0, \frac{1}{3}\right]$ and according to these conditions we obtain the naked singularity as a result of gravitational collapse. Now we give conditions for mass function when the singularity is gravitationally strong.

\section{The Strength of Singularity}

In this section we use the definition which was given in the paper. ${ }^{9}$ The singularity is gravitationally strong if:

$$
\begin{array}{r}
\lim _{\tau \rightarrow 0} \tau^{2} \psi>0, \\
\psi=R_{\alpha \beta} K^{\alpha} K^{\beta}, \\
K^{\alpha}=\frac{d x^{\alpha}}{d \tau},
\end{array}
$$

here $\tau$ - affine parameter, $K^{\alpha}$ is tangent vector to geodesic at the singularity. In the paper ${ }^{5}$ it has been shown that in our case the equation (26) can be written in the following form:

$$
\lim _{\tau \rightarrow 0} \tau^{2} \psi=\frac{1}{4} X_{0}^{2}\left(\varepsilon-\frac{4 \alpha \mu^{2}}{2 \alpha-1} X_{0}^{2 \alpha-1}\right),
$$

where $\varepsilon$ is an arbitrary constant.

Now let us substitute (24) into (27) we obtain:

$$
\frac{1}{4} X_{0}^{2}\left(\varepsilon-\frac{4 \alpha\left(\frac{2\left(C(v)+D(v) r^{1-2 \alpha}\right.}{r^{2}}\right)^{2}}{2 \alpha-1} X_{0}^{2 \alpha-1}\right) .
$$

Now it is not difficult to obtain conditions for the mass function (24) when the singularity is strong:

$$
\begin{array}{r}
\varepsilon \geq 0, \\
\lim _{v \rightarrow 0} \dot{D}(v)=+\infty, \\
\lim _{v \rightarrow 0} \dot{C}(v)=+\infty, \\
\lim _{v \rightarrow 0, r \rightarrow 0} \frac{\dot{C}(v)+\dot{D}(v) r^{1-2 \alpha}}{r^{2}}=+\infty .
\end{array}
$$

Now let us give an explicit example when the singularity is strong. When either $C(v)$ or $D(v)$ in the form $\lambda^{2} v^{\gamma}$, where $\lambda$ is real constant, $\gamma \in(0,1)$.

\section{Conclusion}

In this paper we have considered the endstate of gravitational collapse of generalised Vaidya spacetime in terms of either a black hole or a naked singularity. Also we 
have given conditions for the mass function when the naked singularity is formed and also conditions when the singularity is strong.

The naked singularity formation at the end state of gravitational collapse of spherically symmetric object is one of the opportunities for explanation of Penrose geodesics nature. If the naked singularity was formed in case of gravitational collapse with rotation then we would can explain emergence in the ergosphere from a region inside gravitational radius not only Penrose geodesics but so-called white hole geodesics which was classified in the paper. ${ }^{10}$

\section{Acknowledgments}

The author thanks to professor A.A.Grib for scientific discussion. This work was supported by RFBR, grant 15-02-06818-a and "Dynasty Foundation".

\section{References}

1. A. A. Grib, Yu. V. Pavlov and V. D. Vertogradov, Modern Physics Letters A 29, 14501 (2014).

2. V. D. Vertogradov, Gravitation and Cosmology 21, 171 (2015).

3. A. Papapetrou, in A Random Walk in Relativity and Cosmology (Wiley, New Delhi, 1985).

4. S. P. Joshi, Gravitational Collapse and Spacetime Singularities (Cambridge University Press, Cambridge, 2007), p.273.

5. M. D. Maombi, R. Goswami and S. D. Maharaj, Gravitational collapse of generalised Vaidya spacetime, arXiv:1407.4309.

6. S. M. Wagh and S. D. Maharaj, Naked singularity of the Vaidya-deSitter spacetime and cosmic censorship hypothesis, arXiv:9903083.

7. C. B. Nolan, Phys. Rev. D 60, 024014 (1999).

8. A. Wang and Yu. Wu, Generalized Vaidya solutions, arXiv:9803038.

9. F. J. Tipler, Phys. Lett. A 64, 8 (1977).

10. A. A. Grib and Yu. V. Pavlov, Gravitation and Cosmology 21, 13 (2015). 